\title{
Existence and Uniqueness Results for Fractional Differential Equations with Riemann-Liouville Fractional Integral Boundary Conditions
}

\author{
Mohamed I. Abbas \\ Department of Mathematics and Computer Science, Faculty of Science, Alexandria University, Alexandria 21511, Egypt \\ Correspondence should be addressed to Mohamed I. Abbas; miabbas77@gmail.com
}

Received 12 September 2015; Accepted 17 November 2015

Academic Editor: Jozef Banas

Copyright (C) 2015 Mohamed I. Abbas. This is an open access article distributed under the Creative Commons Attribution License, which permits unrestricted use, distribution, and reproduction in any medium, provided the original work is properly cited.

We prove the existence and uniqueness of solution for fractional differential equations with Riemann-Liouville fractional integral boundary conditions. The first existence and uniqueness result is based on Banach's contraction principle. Moreover, other existence results are also obtained by using the Krasnoselskii fixed point theorem. An example is given to illustrate the main results.

\section{Introduction}

Very recently, fractional differential equations have gained much attention due to extensive applications of these equations in the mathematical modelling of physical, engineering, biological phenomena and viscoelasticity (see, e.g., $[1,2]$ ). There has been a significant development in fractional differential equations. One can see the monographs of Kilbas et al. [3], Miller and Ross [4], Lakshmikantham et al. [5], and Podlubny [6]. In particular, Agarwal et al. [7] establish sufficient conditions for the existence and uniqueness of solutions for various classes of initial and boundary value problem for fractional differential equations and inclusions involving the Caputo fractional derivative in finite dimensional spaces.

Moreover, the theory of boundary value problems with integral boundary conditions for ordinary differential equations arises in different areas of applied mathematics and physics. For example, heat conduction, chemical engineering, underground water flow, thermoelasticity, population dynamics [8], and cellular systems [9] can be reduced to the nonlocal problems with integral boundary conditions. For boundary value problems with integral boundary conditions and comments on their importance, we refer the reader to the papers [10-13] and so forth.

In this paper, we study the existence and uniqueness of solution for fractional differential equations with
Riemann-Liouville fractional integral boundary conditions of the following form:

$$
\begin{aligned}
& { }^{C} D^{q} x(t)=f(t, x(t)), \\
& t \in J:=[0,1], 1<q \leq 2, \\
& \alpha_{1} x(0)+\left.\beta_{1} I^{p} x(t)\right|_{t=0}=\gamma_{1}, \\
& \alpha_{2} x(1)+\left.\beta_{2} I^{p} x(t)\right|_{t=1}=\gamma_{2} \text {, } \\
& 0<p<1,
\end{aligned}
$$

where ${ }^{C} D^{q}$ is the Caputo fractional derivative of order $q, I^{p}$ is the Riemann-Liouville fractional integral of order $p$, and $\alpha_{i}, \beta_{i}, \gamma_{i}(i=1,2)$ are real constants.

Moreover, $f: J \times E \rightarrow E$ is a continuous function satisfying some assumptions that will be specified later and $E$ is a Banach space with norm $\|\cdot\|$.

\section{Preliminaries}

We now gather some definitions and preliminary facts which will be used throughout this paper. Denote by $C(J, E)$ the Banach space of continuous functions $x: J \rightarrow E$, with the usual supremum norm

$$
\|x\|_{\infty}=\sup _{t \in J}\|x(t)\| .
$$


We need some basic definitions and properties $[6,14]$ of fractional calculus which are used in this paper.

Definition 1. The Riemann-Liouville fractional integral of order $q>0$ of the function $h \in L^{1}([a, b])$ is defined as

$$
I^{q} h(t)=\frac{1}{\Gamma(q)} \int_{0}^{t} \frac{h(s)}{(t-s)^{1-q}} d s .
$$

Definition 2. For a function $h$ defined on the interval $[a, b]$, the Caputo fractional order derivative of $h$ is defined by

$$
{ }^{C} D^{q} h(t)=\frac{1}{\Gamma(n-q)} \int_{0}^{t} \frac{h^{(n)}(s)}{(t-s)^{q-n+1}} d s,
$$

where $n=[q]+1$ and $[q]$ denotes the integer part of $q$.

From the definition of the Caputo derivative, the following auxiliary results have been established in [3].

Lemma 3. Let $q>0$; then the differential equation ${ }^{C} D_{t}^{q} h(t)=$ 0 has solutions

$$
h(t)=c_{0}+c_{1} t+c_{2} t^{2}+\cdots+c_{m-1} t^{m-1},
$$

for some $c_{i} \in \mathbb{R}, i=0,1, \ldots, m-1$.

Lemma 4. Let $q>0$; then

$$
I^{q C} D_{t}^{q} h(t)=h(t)+c_{0}+c_{1} t+c_{2} t^{2}+\cdots+c_{m-1} t^{m-1},
$$

for some $c_{i} \in \mathbb{R}, i=0,1, \ldots, m-1, m=-[-q]$.

To study the boundary value problem (1), we first consider the associated linear problem and obtain its solution.

Lemma 5. For a given $\sigma \in C(J, E)$, the unique mild solution of the fractional boundary value problem,

$$
\begin{aligned}
{ }^{C} D_{t}^{q} x(t) & =\sigma(t), \quad 0<q<1, t \in J, \\
\alpha_{1} x(0)+\left.\beta_{1} I^{p} x(t)\right|_{t=0} & =\gamma_{1}, \\
\alpha_{2} x(1)+\left.\beta_{2} I^{p} x(t)\right|_{t=1} & =\gamma_{2},
\end{aligned}
$$

$$
0<p<1
$$

satisfies the following integral equation:

$$
\begin{aligned}
& x(t)=\frac{\gamma_{1}}{\alpha_{1}}+\nu_{1} t\left(\left(\gamma_{2}-\frac{\gamma_{1}}{v_{2} \alpha_{1}}\right)\right. \\
& -\int_{0}^{1}\left(\frac{\alpha_{2}}{\Gamma(q)}(1-s)^{q-1}+\frac{\beta_{2}}{\Gamma(p+q)}(1-s)^{p+q-1}\right) \\
& \cdot \sigma(s) d s)+\frac{1}{\Gamma(q)} \int_{0}^{t}(t-s)^{q-1} \sigma(s) d s
\end{aligned}
$$

where

$$
\begin{aligned}
& v_{1}=\frac{\Gamma(p+2)}{\alpha_{2} \Gamma(p+2)+\beta_{2}}, \\
& v_{2}=\frac{\Gamma(p+1)}{\alpha_{2} \Gamma(p+1)+\beta_{2}} .
\end{aligned}
$$

Proof. By Lemma 4, we reduce (7) to an equivalent integral equation

$$
\begin{aligned}
x(t) & =I^{q} \sigma(t)-c_{0}-c_{1} t \\
& =\frac{1}{\Gamma(q)} \int_{0}^{t}(t-s)^{q-1} \sigma(s) d s-c_{0}-c_{1} t .
\end{aligned}
$$

Then we have

$$
\begin{aligned}
& I^{p} x(t)=\frac{1}{\Gamma(p)} \int_{0}^{t}(t-s)^{p-1} x(s) d s=\frac{1}{\Gamma(p)} \\
& \cdot \int_{0}^{t}(t-s)^{p-1} \\
& \cdot\left(\frac{1}{\Gamma(q)} \int_{0}^{s}(s-\tau)^{q-1} \sigma(\tau) d \tau-c_{0}-c_{1} s\right) d s \\
& =\frac{1}{\Gamma(p) \Gamma(q)} \int_{0}^{t}\left(\int_{\tau}^{t}(t-s)^{p-1}(s-\tau)^{q-1} d s\right) \\
& \cdot \sigma(\tau) d \tau-\frac{c_{0} t^{p}}{\Gamma(p+1)}-\frac{c_{1}}{\Gamma(p)} \int_{0}^{t}(t-s)^{p-1} s d s \\
& =\frac{\mathbf{B}(p, q)}{\Gamma(p) \Gamma(q)} \int_{0}^{t}(t-\tau)^{p+q-1} \sigma(\tau) d \tau-\frac{c_{0} t^{p}}{\Gamma(p+1)} \\
& -\frac{c_{1}}{\Gamma(p)} \int_{0}^{t}(t-s)^{p-1} s d s=\frac{1}{\Gamma(p+q)} \\
& \cdot \int_{0}^{t}(t-\tau)^{p+q-1} \sigma(\tau) d \tau-\frac{c_{0} t^{p}}{\Gamma(p+1)}-\frac{c_{1}}{\Gamma(p)} \\
& \quad \int_{0}^{t}(t-s)^{p-1} s d s,
\end{aligned}
$$

where the integral

$$
\begin{aligned}
& \int_{\tau}^{t}(t-s)^{p-1}(s-\tau)^{q-1} d s \\
& \quad=(t-\tau)^{p+q-1} \int_{0}^{1}(1-z)^{p-1} z^{q-1} d z \\
& \quad=(t-\tau)^{p+q-1} \mathbf{B}(p, q)
\end{aligned}
$$

is evaluated with the help of the substitution $s=\tau+z(t-$ $\tau)$ and the definition of the beta function $\mathbf{B}(p, q)=\int_{0}^{1}(t-$ $s)^{p-1} s^{q-1} d s=\Gamma(p) \Gamma(q) / \Gamma(p+q)$.

Applying the boundary conditions in (7), we have

$-c_{0} \alpha_{1}+\beta_{1} \times 0=\gamma_{1}$, which implies that $c_{0}=-\frac{\gamma_{1}}{\alpha_{1}}$,

$$
\begin{aligned}
& \alpha_{2}\left(\int_{0}^{1} \frac{(1-s)^{q-1}}{\Gamma(q)} \sigma(s) d s-c_{0}-c_{1}\right) \\
& \quad+\beta_{2}\left(\int_{0}^{1} \frac{(1-s)^{p+q-1}}{\Gamma(p+q)} \sigma(s) d s-c_{0} \int_{0}^{1} \frac{(1-s)^{p-1}}{\Gamma(p)} d s\right. \\
& \left.\quad-c_{1} \int_{0}^{1} \frac{(1-s)^{p-1} s}{\Gamma(p)} d s\right)=\gamma_{2} .
\end{aligned}
$$


Using (13) in (14) together with (9), we obtain

$$
\begin{aligned}
c_{1}= & \nu_{1} \int_{0}^{1}\left(\alpha_{2} \frac{(1-s)^{q-1}}{\Gamma(q)}+\beta_{2} \frac{(1-s)^{p+q-1}}{\Gamma(p+q)}\right) \sigma(s) d s \\
& -\nu_{1}\left(\gamma_{2}-\frac{\gamma_{1}}{v_{2} \alpha_{1}}\right) .
\end{aligned}
$$

Substituting the values of $c_{0}$ and $c_{1}$ in (10), we get (8). This completes the proof.

Lemma 6 (Arzelà-Ascoli, [15]). If a sequence $\left\{x_{n}\right\}$ in a compact subset of $X$ is uniformly bounded and equicontinuous, then it has a uniformly convergent subsequence.

Lemma 7 (Krasnoselskii, [15]). Let $\Omega$ be a closed convex and nonempty subset of a Banach space $X$. Let $A$ and $B$ be two operators such that

(i) $A x+B y \in \Omega$, wherever $x, y \in \Omega$;

(ii) $A$ is compact and continuous;

(iii) $B$ is a contraction mapping.

Then there exists $z \in \Omega$ such that $z=A z+B z$.

\section{Main Results}

In view of Lemma 5 , we define the operator $T: C(J, E) \rightarrow$ $C(J, E)$ as follows:

$$
\begin{aligned}
& (T x)(t)=\frac{\gamma_{1}}{\alpha_{1}}+v_{1} t\left[\left(\gamma_{2}-\frac{\gamma_{1}}{v_{2} \alpha_{1}}\right)\right. \\
& -\int_{0}^{1}\left(\frac{\alpha_{2}}{\Gamma(q)}(1-s)^{q-1}+\frac{\beta_{2}}{\Gamma(p+q)}(1-s)^{p+q-1}\right) \\
& \cdot f(s, x(s)) d s]+\frac{1}{\Gamma(q)} \int_{0}^{t}(t-s)^{q-1} \\
& \cdot f(s, x(s)) d s, \quad t \in J .
\end{aligned}
$$

For the forthcoming analysis we impose suitable conditions on the functions involved in the boundary value problem (1). Namely, we assume the following:

(H1) The function $f: J \times E \rightarrow E$ is continuous and satisfies the following Lipschitz condition with constant $k>0$ :

$$
\|f(t, x)-f(t, y)\| \leq k\|x-y\|, \quad x, y \in E, t \in J .
$$

(H2) Let $d$ and $r$ be two nonnegative real numbers such that $0<d<1$ and

$$
\begin{aligned}
& k\left(\frac{1+\left|\nu_{1}\right|\left|\alpha_{2}\right|}{\Gamma(q+1)}+\frac{\left|\nu_{1}\right|\left|\beta_{2}\right|}{\Gamma(p+q+1)}\right)<d, \\
& M\left(\frac{1+\left|\nu_{1}\right|\left|\alpha_{2}\right|}{\Gamma(q+1)}+\frac{\left|\nu_{1}\right|\left|\beta_{2}\right|}{\Gamma(p+q+1)}\right) \\
& \quad+\left|\nu_{1}\right|\left(\left|\gamma_{2}\right|+\frac{\left|\gamma_{1}\right|}{\left|\nu_{2}\right|\left|\alpha_{1}\right|}\right)+\frac{\left|\gamma_{1}\right|}{\left|\alpha_{1}\right|} \leq(1-d) r,
\end{aligned}
$$

Our first result is based on Banach's contraction principle.

Theorem 8. Assume that conditions (H1) and (H2) are satisfied. Then the boundary value problem (1) has a unique solution in $C(J, E)$.

Proof. We need to prove that the operator $T$ has a fixed point on the set $B_{r}=\{x \in E:\|x\| \leq r\}$. For $x \in B_{r}$, we have

$$
\begin{aligned}
& \|(T x)(t)\| \leq \frac{\left|\gamma_{1}\right|}{\left|\alpha_{1}\right|}+\left|\nu_{1}\right|\left[\left(\left|\gamma_{2}\right|+\frac{\left|\gamma_{1}\right|}{\left|\nu_{2}\right|\left|\alpha_{1}\right|}\right)\right. \\
& +\int_{0}^{1}\left(\frac{\left|\alpha_{2}\right|}{\Gamma(q)}(1-s)^{q-1}+\frac{\left|\beta_{2}\right|}{\Gamma(p+q)}(1-s)^{p+q-1}\right) \\
& \text { - }\|f(s, x(s))-f(s, 0)\| d s \\
& +\int_{0}^{1}\left(\frac{\left|\alpha_{2}\right|}{\Gamma(q)}(1-s)^{q-1}+\frac{\left|\beta_{2}\right|}{\Gamma(p+q)}(1-s)^{p+q-1}\right) \\
& \cdot\|f(s, 0)\| d s]+\frac{1}{\Gamma(q)} \int_{0}^{t}(t-s)^{q-1} \\
& \cdot\|f(s, x(s))-f(s, 0)\| d s+\frac{1}{\Gamma(q)} \int_{0}^{t}(t \\
& -s)^{q-1}\|f(s, 0)\| d s \leq \frac{\left|\gamma_{1}\right|}{\left|\alpha_{1}\right|}+\left|\nu_{1}\right|\left[\left(\left|\gamma_{2}\right|\right.\right. \\
& \left.+\frac{\left|\gamma_{1}\right|}{\left|\nu_{2}\right|\left|\alpha_{1}\right|}\right)+k\|x\| \int_{0}^{1}\left(\frac{\left|\alpha_{2}\right|}{\Gamma(q)}(1-s)^{q-1}\right. \\
& \left.+\frac{\left|\beta_{2}\right|}{\Gamma(p+q)}(1-s)^{p+q-1}\right) d s \\
& +M \int_{0}^{1}\left(\frac{\left|\alpha_{2}\right|}{\Gamma(q)}(1-s)^{q-1}\right. \\
& \left.\left.+\frac{\left|\beta_{2}\right|}{\Gamma(p+q)}(1-s)^{p+q-1}\right) d s\right]+\frac{k\|x\|}{\Gamma(q)} \int_{0}^{t}(t \\
& -s)^{q-1} d s+\frac{M}{\Gamma(q)} \int_{0}^{t}(t-s)^{q-1} d s \leq \frac{\left|\gamma_{1}\right|}{\left|\alpha_{1}\right|} \\
& +\left|v_{1}\right|\left[\left(\left|\gamma_{2}\right|+\frac{\left|\gamma_{1}\right|}{\left|\nu_{2}\right|\left|\alpha_{1}\right|}\right)+k\left(\frac{\left|\alpha_{2}\right|}{\Gamma(q+1)}\right.\right. \\
& \left.+\frac{\left|\beta_{2}\right|}{\Gamma(p+q+1)}\right) r+M\left(\frac{\left|\alpha_{2}\right|}{\Gamma(q+1)}\right. \\
& \left.\left.+\frac{\left|\beta_{2}\right|}{\Gamma(p+q+1)}\right)\right]+\frac{k r}{\Gamma(q+1)}+\frac{M}{\Gamma(p+q+1)} \\
& =k\left(\frac{1+\left|\nu_{1}\right|\left|\alpha_{2}\right|}{\Gamma(q+1)}+\frac{\left|v_{1}\right|\left|\beta_{2}\right|}{\Gamma(p+q+1)}\right) r
\end{aligned}
$$




$$
\begin{aligned}
& +M\left(\frac{1+\left|\nu_{1}\right|\left|\alpha_{2}\right|}{\Gamma(q+1)}+\frac{\left|\nu_{1}\right|\left|\beta_{2}\right|}{\Gamma(p+q+1)}\right)+\left|\nu_{1}\right|\left(\left|\gamma_{2}\right|\right. \\
& \left.+\frac{\left|\gamma_{1}\right|}{\left|\nu_{2}\right|\left|\alpha_{1}\right|}\right)+\frac{\left|\gamma_{1}\right|}{\left|\alpha_{1}\right|} .
\end{aligned}
$$

Therefore,

$$
\|T x\| \leq d r+(1-d) r
$$

which implies that $T B_{r} \subset B_{r}$. Hence, $T$ maps $B_{r}$ into itself.

Now, we will prove that $T$ is a contraction mapping on $B_{r}$. For $x, y \in B_{r}$, we have

$$
\begin{aligned}
& \|(T x)(t)-(T y)(t)\| \leq\left|v_{1}\right| \\
& \cdot \int_{0}^{1}\left(\frac{\left|\alpha_{2}\right|}{\Gamma(q)}(1-s)^{q-1}+\frac{\left|\beta_{2}\right|}{\Gamma(p+q)}(1-s)^{p+q-1}\right) \\
& \cdot\|f(s, x(s))-f(s, y(s))\| d s+\frac{1}{\Gamma(q)} \\
& \cdot \int_{0}^{t}(t-s)^{q-1}\|f(s, x(s))-f(s, y(s))\| d s \\
& \quad \leq k\left(\frac{1+\left|v_{1}\right|\left|\alpha_{2}\right|}{\Gamma(q+1)}+\frac{\left|v_{1}\right|\left|\beta_{2}\right|}{\Gamma(p+q+1)}\right)\|x-y\| .
\end{aligned}
$$

Therefore,

$$
\|T x-T y\| \leq d\|x-y\| .
$$

Hence, the operator $T$ is a contraction. Then $T$ has a unique fixed point which is a solution of the boundary value problem (1).

The following result is based on the Krasnoselskii fixed point theorem. To apply this theorem, we need the following hypothesis:

(H3) There exist $\phi(t) \in L^{1}\left(J, \mathbb{R}_{+}\right)$such that

$$
\|f(t, x)\| \leq \phi(t), \quad \forall(t, x) \in J \times E .
$$

Theorem 9. Assume that conditions (H1) and (H3) are satisfied. Then the boundary value problem (1) has at least a solution in $C(J, E)$, provided that

$$
l:=k\left|\nu_{1}\right|\left(\frac{\left|\alpha_{2}\right|}{\Gamma(q+1)}+\frac{\left|\beta_{2}\right|}{\Gamma(p+q+1)}\right)<1 .
$$

Proof. Letting $\phi^{*}:=\sup _{t \in J} \phi(t)$, we fix

$$
\begin{aligned}
\rho \geq & \frac{\left|\gamma_{1}\right|}{\left|\alpha_{1}\right|}+\left|\nu_{1}\right|\left(\left|\gamma_{2}\right|+\frac{\left|\gamma_{1}\right|}{\left|\nu_{2}\right|\left|\alpha_{1}\right|}\right) \\
& +\left(\frac{1+\left|\nu_{1}\right|\left|\alpha_{2}\right|}{\Gamma(q+1)}+\frac{\left|\nu_{1}\right|\left|\beta_{2}\right|}{\Gamma(p+q+1)}\right) \phi^{*} .
\end{aligned}
$$

On $B_{\rho}=\{x \in E:\|x\| \leq \rho\}$, we define the two operators $R$ and $S$ as follows:

$$
\begin{aligned}
& (R x)(t)=\frac{\gamma_{1}}{\alpha_{1}}+\nu_{1} t\left[\left(\gamma_{2}-\frac{\gamma_{1}}{\nu_{2} \alpha_{1}}\right)\right. \\
& -\int_{0}^{1}\left(\alpha_{2} \frac{(1-s)^{q-1}}{\Gamma(q)}+\beta_{2} \frac{(1-s)^{p+q-1}}{\Gamma(p+q)}\right) \\
& \cdot f(s, x(s)) d s], \quad t \in J, \\
& (S x)(t)=\frac{1}{\Gamma(q)} \int_{0}^{t}(t-s)^{q-1} f(s, x(s)) d s, \quad t \in J .
\end{aligned}
$$

For $x, y \in B_{\rho}$, we have

$$
\begin{aligned}
& \|R x+S y\| \leq \frac{\left|\gamma_{1}\right|}{\left|\alpha_{1}\right|}+\left|v_{1}\right|\left[\left(\left|\gamma_{2}\right|+\frac{\left|\gamma_{1}\right|}{\left|\nu_{2}\right|\left|\alpha_{1}\right|}\right)\right. \\
& +\int_{0}^{1}\left(\frac{\left|\alpha_{2}\right|}{\Gamma(q)}(1-s)^{q-1}+\frac{\left|\beta_{2}\right|}{\Gamma(p+q)}(1-s)^{p+q-1}\right) \\
& \cdot\|f(s, x(s))\| d s]+\frac{1}{\Gamma(q)} \int_{0}^{t}(t-s)^{q-1} \\
& \cdot\|f(s, y(s))\| d s \leq \frac{\left|\gamma_{1}\right|}{\left|\alpha_{1}\right|}+\left|v_{1}\right|\left[\left(\left|\gamma_{2}\right|\right.\right. \\
& \left.+\frac{\left|\gamma_{1}\right|}{\left|\nu_{2}\right|\left|\alpha_{1}\right|}\right)+\int_{0}^{1}\left(\frac{\left|\alpha_{2}\right|}{\Gamma(q)}(1-s)^{q-1}\right. \\
& \left.\left.+\frac{\left|\beta_{2}\right|}{\Gamma(p+q)}(1-s)^{p+q-1}\right) \phi(s) d s\right]+\frac{1}{\Gamma(q)} \int_{0}^{t}(t \\
& -s)^{q-1} \phi(s) d s \leq \frac{\left|\gamma_{1}\right|}{\left|\alpha_{1}\right|}+\left|\nu_{1}\right|\left[\left(\left|\gamma_{2}\right|+\frac{\left|\gamma_{1}\right|}{\left|\nu_{2}\right|\left|\alpha_{1}\right|}\right)\right. \\
& +\phi^{*} \int_{0}^{1}\left(\frac{\left|\alpha_{2}\right|}{\Gamma(q)}(1-s)^{q-1}\right. \\
& \left.\left.+\frac{\left|\beta_{2}\right|}{\Gamma(p+q)}(1-s)^{p+q-1}\right) d s\right]+\phi^{*} \frac{1}{\Gamma(q)} \int_{0}^{t}(t \\
& -s)^{q-1} d s \leq \frac{\left|\gamma_{1}\right|}{\left|\alpha_{1}\right|}+\left|\nu_{1}\right|\left[\left(\left|\gamma_{2}\right|+\frac{\left|\gamma_{1}\right|}{\left|\nu_{2}\right|\left|\alpha_{1}\right|}\right)\right. \\
& \left.+\phi^{*}\left(\frac{\left|\alpha_{2}\right|}{\Gamma(q+1)}+\frac{\left|\beta_{2}\right|}{\Gamma(p+q+1)}\right)\right]+\frac{\phi^{*}}{\Gamma(q+1)} \\
& =\frac{\left|\gamma_{1}\right|}{\left|\alpha_{1}\right|}+\left|\nu_{1}\right|\left(\left|\gamma_{2}\right|+\frac{\left|\gamma_{1}\right|}{\left|\nu_{2}\right|\left|\alpha_{1}\right|}\right)+\left(\frac{1+\left|\nu_{1}\right|\left|\alpha_{2}\right|}{\Gamma(q+1)}\right. \\
& \left.+\frac{\left|v_{1}\right|\left|\beta_{2}\right|}{\Gamma(p+q+1)}\right) \phi^{*} \leq \rho \text {. }
\end{aligned}
$$

Hence, $R x+S y \in B_{\rho}$. 
On the other hand, from (H1) together with (24), it is easy to see that

$$
\|R x-R y\| \leq l\|x-y\|,
$$

and since $l<1$, then $R$ is a contraction mapping.

Moreover, continuity of $f$ implies that the operator $S$ is continuous. Also, $S$ is uniformly bounded on $B_{\rho}$ and

$$
\|S x\| \leq \frac{\phi^{*}}{\Gamma(q+1)} .
$$

Now we prove the compactness of the operator $S$.

In view of (H1) we define $L:=\sup _{(t, x) \in J \times B_{\rho}} f(t, x)$ and consequently we have for $t_{1}, t_{2} \in J, t_{1}<t_{2}$, and $x \in B_{\rho}$

$$
\begin{aligned}
& \left\|(S x)\left(t_{2}\right)-(S x)\left(t_{1}\right)\right\|=\sup _{(t, x) \in J \times B_{\rho}} \mid \frac{1}{\Gamma(q)} \\
& \cdot \int_{0}^{t_{2}}\left[\left(t_{2}-s\right)^{q-1}-\left(t_{1}-s\right)^{q-1}\right] f(s, x(s)) d s \\
& +\frac{1}{\Gamma(q)} \int_{t_{1}}^{t_{2}}\left(t_{1}-s\right)^{q-1} f(s, x(s)) d s\left|\leq \frac{L}{\Gamma(q)}\right| t_{2}^{q} \\
& -t_{1}^{q} \mid,
\end{aligned}
$$

which is independent of $x$ and tends to zero as $t_{2}-t_{1} \rightarrow 0$. Thus, $S$ is equicontinuous. Hence, by the Arzelà-Ascoli Theorem (Lemma 6), $S$ is compact on $B_{\rho}$. Thus, all the assumptions of Lemma 7 are satisfied. So the conclusion of the Krasnoselskii fixed point theorem implies that the boundary value problem (1) has at least one solution on $J$. This completes the proof of Theorem 9 .

\section{An Example}

Consider the following fractional boundary value problem:

$$
\begin{aligned}
{ }^{C} D_{t}^{4 / 3} x(t) & =\frac{1}{e^{t+2}}\left(\frac{|x(t)|}{|x(t)|+1}\right), \\
t \in[0,1], & \\
x(0)+\left.I^{1 / 3} x(t)\right|_{t=0} & =\frac{1}{2}, \\
\frac{1}{2} x(1)+\left.\frac{1}{3} I^{1 / 3} x(t)\right|_{t=1} & =2,
\end{aligned}
$$

where $q=4 / 3, p=1 / 3, \alpha_{1}=1, \alpha_{2}=1 / 2, \beta_{1}=1, \beta_{2}=1 / 3$, $\gamma_{1}=1 / 2, \gamma_{2}=2$, and $\nu_{1} \simeq 1.282114, \nu_{2} \simeq 1.145105$.

Here, $f:[0,1] \times \mathbb{R} \rightarrow \mathbb{R}$ is given by

$$
f(t, x)=\frac{1}{e^{t+2}}\left(\frac{|x|}{|x|+1}\right), \quad \forall(t, x) \in[0,1] \times \mathbb{R} .
$$

Then we have

$$
\|f(t, x)-f(t, y)\| \leq \frac{1}{e^{2}}\|x-y\| .
$$

Therefore, $(\mathrm{H} 1)$ is satisfied with $k=1 / e^{2} \simeq 0.135335$.
Moreover

$$
\begin{aligned}
\|f(t, x)\| & =\left\|\frac{1}{e^{t+2}}\left(\frac{|x|}{|x|+1}\right)\right\| \leq \frac{1}{e^{t+2}}\left\|\frac{|x|}{|x|+1}\right\| \\
& \leq \frac{1}{e^{t+2}} .
\end{aligned}
$$

Therefore, $\phi(t)=1 / e^{t+2}, \forall t \in[0,1]$, and $\phi^{*}=1 / e^{2} \simeq$ 0.135335 .

Also, we get $M=\sup _{t \in J}|f(t, 0)|=1 / e^{2} \simeq 0.135335$.

Finally, simple calculations give

$$
\begin{aligned}
& k\left(\frac{1+\left|\nu_{1}\right|\left|\alpha_{2}\right|}{\Gamma(q+1)}+\frac{\left|\nu_{1}\right|\left|\beta_{2}\right|}{\Gamma(p+q+1)}\right) \simeq 0.224974<1, \\
& l=k\left|\nu_{1}\right|\left(\frac{\left|\alpha_{2}\right|}{\Gamma(q+1)}+\frac{\left|\beta_{2}\right|}{\Gamma(p+q+1)}\right) \simeq 0.130704 \\
& \quad<1 .
\end{aligned}
$$

Clearly, all the assumptions of Theorems 8 and 9 are satisfied. So there exists at least one solution of the boundary value problem (31) on $[0,1]$.

\section{Conclusion}

In this paper, we study the existence and uniqueness for fractional order differential equations with Riemann-Liouville integral boundary conditions (1) in Banach spaces. Existence and uniqueness results of solutions are established by virtue of fractional calculus, Banach's contraction principle, and the Krasnoselskii fixed point theorem. As applications, an example is presented to illustrate the main results.

\section{Conflict of Interests}

The author declares that there is no conflict of interests regarding the publication of this paper.

\section{References}

[1] R. L. Bagley and P. J. Torvik, "A theoretical basis for the application of fractional calculus to viscoelasticity," Journal of Rheology, vol. 27, no. 3, pp. 201-210, 1983.

[2] K. Diethelm and A. D. Freed, "On the solution of nonlinear fractional order differential equations used in the modelling of viscoplasticity," in Scientific Computing in Chemical Engineering II-Computational Fluid Dynamics and Molecular Properties, F. Keil, W. Mackens, and H. Voss, Eds., pp. 217-224, Springer, Heidelberg, Germany, 1999.

[3] A. A. Kilbas, H. M. Srivastava, and J. J. Trujillo, Theory and Applications of Fractional Differential Equations, vol. 204 of North-Holland Mathematics Studies, Elsevier, Amsterdam, The Netherlands, 2006.

[4] K. S. Miller and B. Ross, An Introduction to the Fractional Calculus and Differential Equations, John Wiley \& Sons, New York, NY, USA, 1993.

[5] V. Lakshmikantham, S. Leela, and J. V. Devi, Theory of Fractional Dynamic Systems, Cambridge Scientific Publishers, 2009.

[6] I. Podlubny, Fractional Differential Equations, Academic Press, San Diego, Calif, USA, 1999. 
[7] R. P. Agarwal, M. Benchohra, and S. Hamani, "A survey on existence results for boundary value problems of nonlinear fractional differential equations and inclusions," Acta Applicandae Mathematicae, vol. 109, no. 3, pp. 973-1033, 2010.

[8] K. W. Blayneh, "Analysis of age structured host-parasitoid model," Far East Journal of Dynamical Systems, vol. 4, pp. 125145, 2002.

[9] G. Adomian and G. E. Adomian, "Cellular systems and aging models," Computers \& Mathematics with Applications, vol. 11, no. 1-3, pp. 283-291, 1985.

[10] B. Ahmad and S. K. Ntouyas, "Fractional differential inclusions with fractional separated boundary conditions," Fractional Calculus and Applied Analysis, vol. 15, no. 3, pp. 362-382, 2012.

[11] B. Ahmad and J. J. Nieto, "Existence results for nonlinear boundary value problems of fractional integrodifferential equations with integral boundary conditions," Boundary Value Problems, vol. 2009, Article ID 708576, 11 pages, 2009.

[12] B. Ahmad and J. J. Nieto, "Riemann-Liouville fractional integrodifferential equations with frac tional nonlocal integral boundary conditions," Boundary Value Problems, vol. 2011, article 36, 2011.

[13] M. Benchohra, J. R. Graef, and S. Hamani, "Existence results for boundary value problems with non-linear fractional differential equations," Applicable Analysis, vol. 87, no. 7, pp. 851-863, 2008.

[14] S. G. Samko, A. A. Kilbas, and O. I. Marichev, Fractional Integrals and Derivatives: Theory and Applications, Gordon and Breach Science Publishers, Englewood Cliffs, NJ, USA, 1993.

[15] D. R. Smart, Fixed Point Theorems, Cambridge University Press, 1980. 


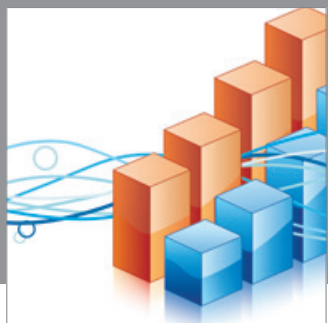

Advances in

Operations Research

mansans

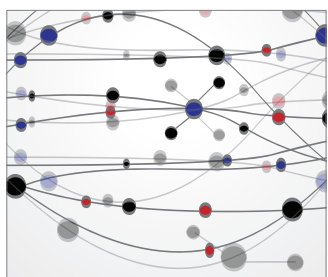

The Scientific World Journal
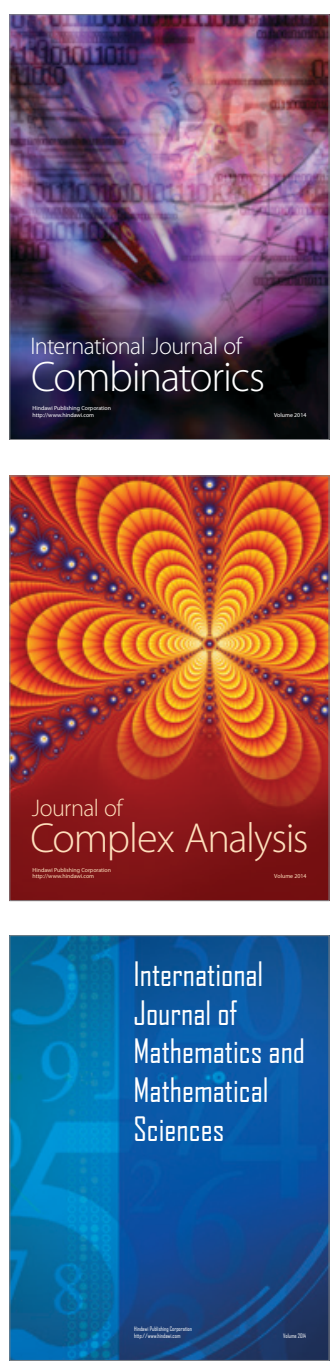
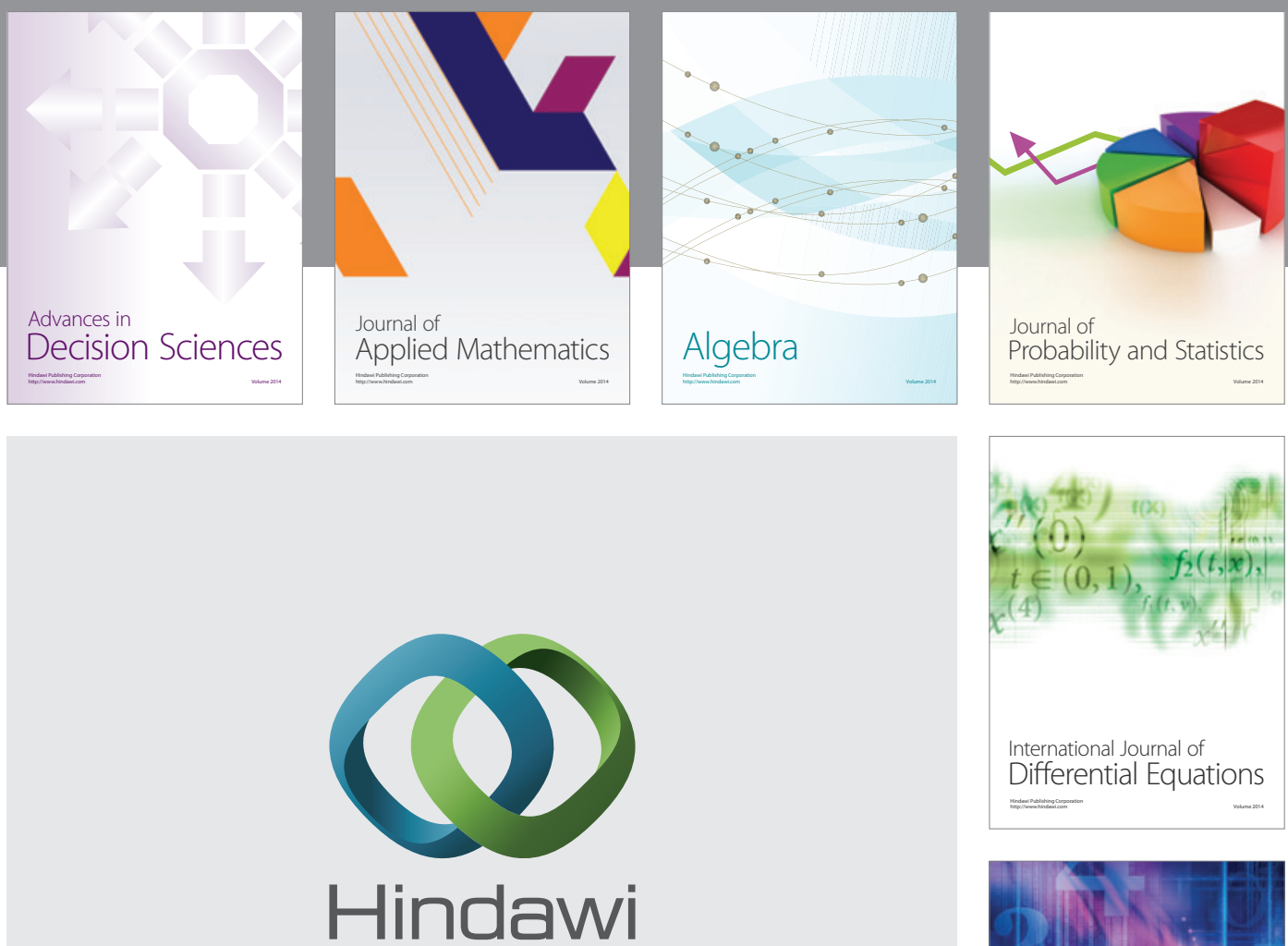

Submit your manuscripts at http://www.hindawi.com
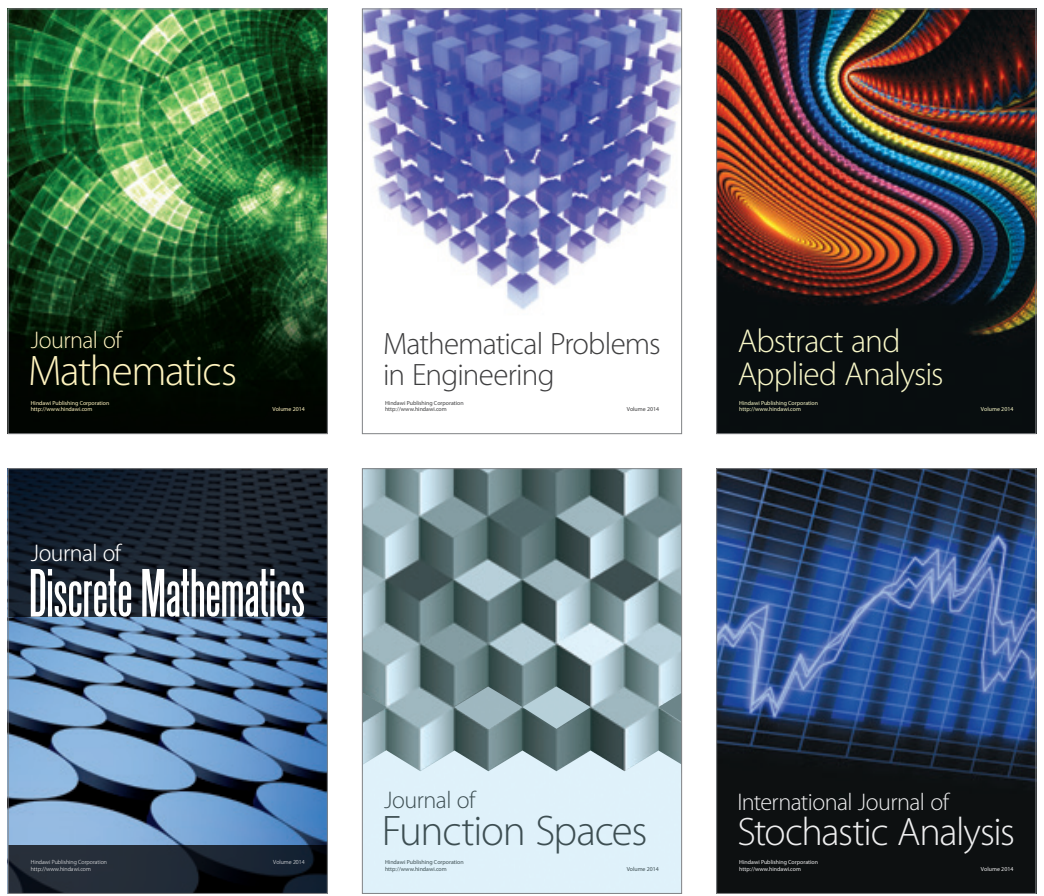

Journal of

Function Spaces

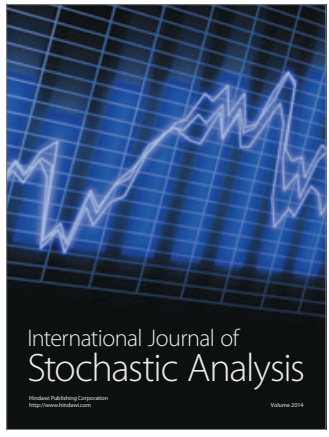

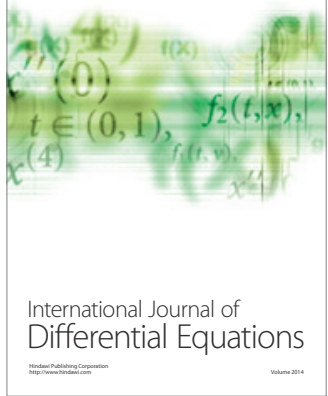
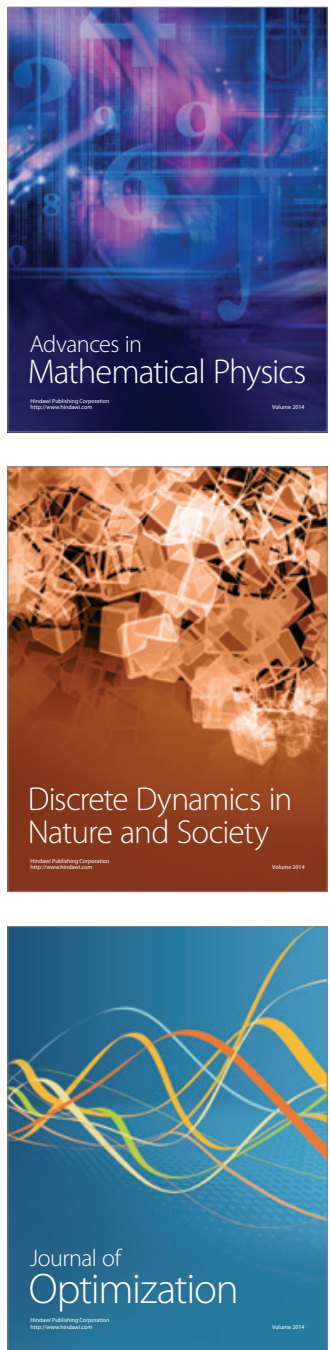